Please do not remove this page

RMIT

UNIVERSITY

\title{
SAW atomization application on inhaled pulmonary drug delivery
}

Qi, Aisha; Friend, James; Yeo, Leslie

https://researchrepository.rmit.edu.au/esploro/outputs/9921858214401341/filesAndLinks?institution=61RMIT_INST\&index=null

Qi, A., Friend, J., \& Yeo, L. (2008). SAW atomization application on inhaled pulmonary drug delivery. Proceedings of SPIE Conference on Biomedical Applications of Micro- and Nanoengineering IV and Complex Systems Vol. 7270, 10-12 December 2008,Melbourne, VIC, 1-10.

https://doi.org/10.1117/12.813983

Document Version: Published Version

Published Version: https://doi.org/10.1117/12.813983

Repository homepage: https://researchrepository.rmit.edu.au

(C) 2008 SPIE

Downloaded On 2023/04/27 01:30:24 +1000

Please do not remove this page 


\title{
SAW Atomization Application on Inhaled Pulmonary Drug Delivery
}

\author{
Aisha Qi, James Friend, Leslie Yeo \\ Micro/Nanophysics Research Laboratory, \\ Monash University, Clayton, VIC 3800, Australia.
}

\begin{abstract}
Pulmonary drug delivery transports the drug formulations directly to the respiratory tract in the form of inhaled particles or droplets. Because of the direct target treatment, it has significant advantages in the treatment of respiratory diseases, for example asthma. However, it is difficult to produce monodispersed particles/droplets in the 1-10 micron range, which is necessary for deposition in the targeted lung area or lower respiratory airways, in a controllable fashion. We demonstrate the use of surface acoustic waves (SAWs) as an efficient method for the generation of monodispersed micron dimension aerosols for the treatment of asthma. SAWs are ten nanometer order amplitude electroacoustic waves generated by applying an oscillating electric field to an interdigital transducer patterned on a piezoelectric substrate. The acoustic energy in the waves induces atomization of the working fluid, which contains a model drug, albuterol. Laser diffraction techniques employed to characterize the aerosols revealed mean diameter of the aerosol was around 3-4 $\mu \mathrm{m}$. Parallel experiments employing a one-stage (glass) twin impinger as a lung model demonstrated a nearly $80 \%$ of atomized drug aerosol was deposited in the lung. The aerosol size distribution is relatively independent of the SAW frequency, which is consistent with our predictive scaling theory which accounts for the dominant balance between viscous and capillary stresses. Moreover, only $1-3 \mathrm{~W}$ powers consumption of SAW atomization suggests that the SAW atomizer can be miniaturized into dimensions commensurate with portable consumer devices.
\end{abstract}

Keywords: Drug Delivery, Surface acoustic wave, SAW, atomization, microfluid

\section{INTRODUCTION}

For the treatment of local lung/chest abnormalities, pulmonary drug delivery transports the drugs to the target area by inhalation, providing an advantage over other drug delivery techniques such as oral and parenteral. Because of the direct target treatment, the inhaled drugs are able to react rapidly at the infected local area with reduced doses and also cause less systemic side effects. ${ }^{1}$

Asthma is believed to be a chronic inflammatory disorder that is associated with an excessive number of lymphocytes and eosinophil cells in the airway walls. ${ }^{2,3}$ This airway inflammation narrows the respiratory tract and causes airflow blockage or obstruction during the human respiration process. In order to ensure the drug particles take effect appropriately, the optimal deposition area of inhaled drugs is the main concern of every inhaled pulmonary therapy for asthma. Once, there were debates on whether the inflammation occurred in the central or narrower airways, ${ }^{3}$ until a number of studies reported that these inflammation related cells, for example, eosinophil cells, were found in every part of the lung area, from the bronchi to the alveoli, when asthma occurred. ${ }^{4-6}$ Therefore, a drug delivery system which enable the produced particles/aerosol be deposited throughout the respiratory tract, from bronchi to alveoli, is highly recommended. ${ }^{3}$

Among the many pulmonary drug administrations methods, jet nebulizers and ultrasonic nebulizers, producing particles/aerosol in different fashions, are the ones that have been most frequently studied and employed in the market. ${ }^{7,8}$ Jet nebulizers generate particles/droplets by means of forcing the pressurized gas through an orifice from a narrow capillary tube, which is embedded in a reservoir containing drug solutions. The original form of jet nebulizers can be traced back to centuries ago, and they are still be widely used today but with more sophisticated forms. However, jet nebulizers have poor performances in producing monodispersed particles/aerosol within required size distribution, and the number of droplets that can pass through the nozzle in a short time is limited, results in a low efficiency.

Biomedical Applications of Micro- and Nanoengineering IV and Complex Systems, edited by Dan V. Nicolau, Guy Metcalfe Proc. of SPIE Vol. 7270, 72700S · ( 2008 SPIE · CCC code: 1605-7422/08/\$18 · doi: 10.1117/12.813983 
Ultrasonic nebulizers contain a piezoelectric element, which is powered by an alternating electric current at a frequency normally between $1 \mathrm{MHz}-3 \mathrm{MHz}$. Drug solutions are usually filled into a reservoir which is placed on the piezoelectric element. Under a sufficient power supply the piezoelectric element, vibrating in a thickness piston mode, is able to induce capillary destabilization of the drug solutions, eventually breaking up the capillary wave and generating the drug aerosol. This is known as ultrasonic atomization.

However, ultrasonic nebulizers also have a number of deficiencies, the need to be miniaturized, high electrical power requirements $(10 \mathrm{~W})$, and most importantly, the precipitated drug loss in the reservoir due to the evaporation of solvents. ${ }^{8}$ Despite these flaws, ultrasonic atomizers are still the most popular nebulizers in the market of asthma treatment, because of a great demand from number of asthma patients, who require the rapid action of drugs in the local area that only the atomization based inhalation therapy can provide. As far as the disadvantages described above in existence, in addition with the high standard requirements of pulmonary administration, the efforts that have been putting into developing new techniques to produce the required aerosol for the inhalation therapy have never stopped. In recent years, a new technology has been developed. Electrohydrodynamic atomization, also called electrospray, becomes an interest in the field of producing monodispersed particels/aerosol for medical purposes. Electro-hydrodynamic atomization applies the electric field on a nozzle, to where a liquid is supplied. The electric stress overcomes the surface tension of the liquid and in turn forms the jet which breaks up into microsized aerosols. ${ }^{9,10}$ This technology has been shown to be able to generate controllable aerosol in the range of 2-3.5 $\mu \mathrm{m}$. However, the requirement of an electrical charge in the kilo-volts range $^{11}$ hinders this technology from being commercialized in the short term.

In this paper, we propose another promising technology, Surface Acoustic Wave (SAW) induced atomization, to be applied on the pulmonary drug administration for a potential asthma treatment. SAW atomizers efficiently generate monodispersed aerosol with controllable sizes, and more importantly, only require $1-3 \mathrm{~W}$ power input. This enables the SAW atomizer be driven by a small scale circuit and to be miniaturized to portable dimensions that are much smaller than other competitors.

SAW is a transverse-axial elliptical electroacoustic acoustic wave with nanometer-order amplitude. It traverses the single-crystal piezoelectric material surface with its amplitude decaying as the substrate depth increases. Even though it has been employed in the telecommunication industry for RF signal processing and filtering for over four decades, ${ }^{12,13}$ SAWs had not been employed to produce aerosol with micron or submicron size particles until the last ten years, when the researches showed its promising ability in atomization. ${ }^{14,15}$

An illustration of this SAW device is shown in Fig. 1(a). A pair of aluminium-chromium interdigital transducers (IDTs) was sputtered (Hummer Triple-target Sputter System) onto a $128^{\circ}$ Y cut X propagation Lithium Niobite $\left(\mathrm{LiNbO}_{3}\right)$ piezoelectric substrate surface. The AC power is supplied through the IDT electrodes and induces a Rayleigh wave, as shown in Fig. 1(b), which propagates along the substrate. Compared to other atomizers, the SAW atomizer runs at much higher frequencies, normally 5-500 MHz. Under the sufficient electrical excitation, the displacement amplitude of the $\mathrm{LiNbO}_{3}$ substrate surface is only approximately $10 \mathrm{~nm}$ in a single period. However, because of the high excitation frequency, for example, from a $10 \mathrm{MHz} \mathrm{SAW}$ device, the acceleration can approach $10^{7} \mathrm{~m} / \mathrm{s}^{2}$ with power requirement of only a few watts. Once a liquid is placed on this surface, SAWs with this huge acceleration propagate into the liquid and can subsequently induce the violent vibration of the drop. When the capillary destabilization of the drop is sufficient, capillary breakup occurs, inducing atomization.

A SAW, which is a type of Rayleigh wave, has different propagation speeds when traveling across different media, for example, on the piezoelectric substrate or in the fluid. When a SAW travels across the piezoelectric substrate and meets the liquid which is placed upon the substrate, the varied propagation speeds cause the SAW to leak into the liquid with an angle known as Rayleigh angle, the value of which is given by $\sin ^{-1}\left(\theta_{l} / \theta_{R}\right)$, where $\theta_{l}$ and $\theta_{R}$ are the propagation speed of Rayleigh wave and the acoustic wave in the liquid, respectively. Thus, as shown in Fig. 1(b), the horizontal component of the excitation becomes the source of a phenomenon known as $S A W$ streaming ${ }^{16,17}$ while the vertical component induces the capillary destabilization and breakup. Our early research ${ }^{18}$ demonstrated that the atomized aerosol diameter, which is closely associated with the capillary wavelength, was largely determined by the competition between the liquid's surface tension and either the viscosity or the inertial force. Theoretically, the diameters of the aerosols are in the range of 1-10 $\mu \mathrm{m}$, which 

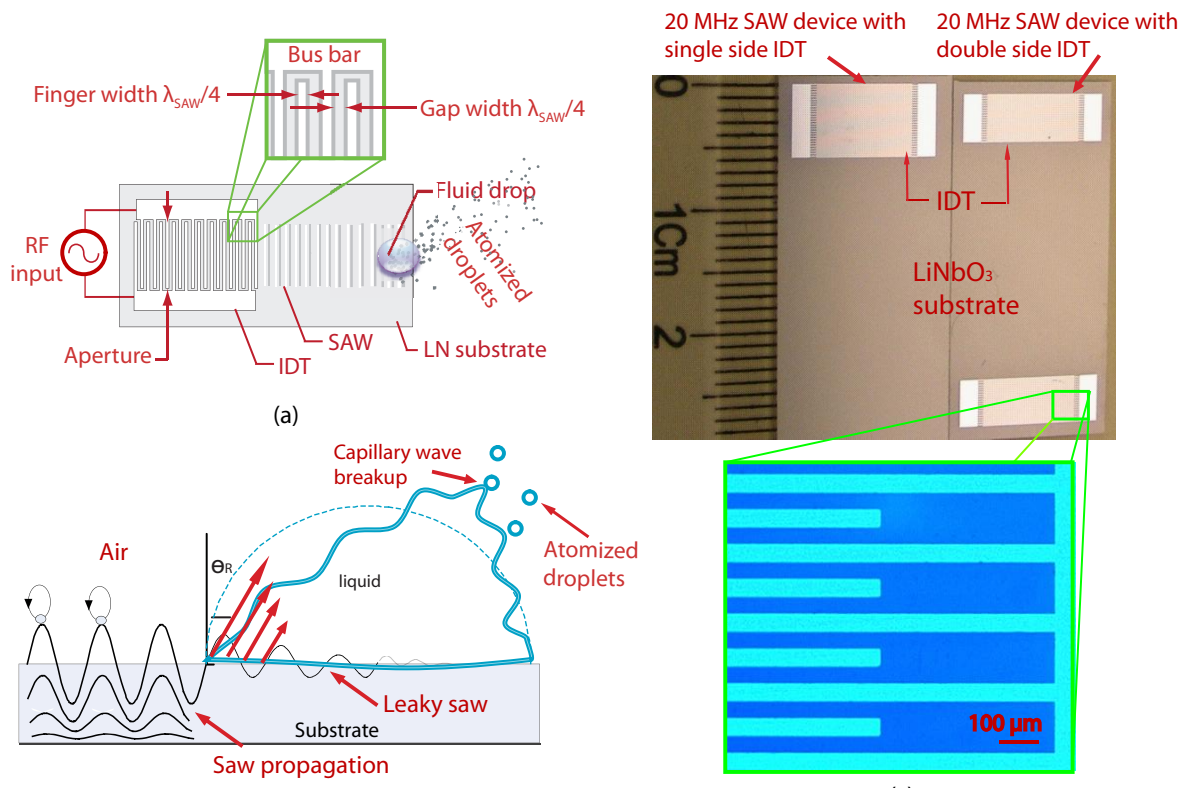

(a)

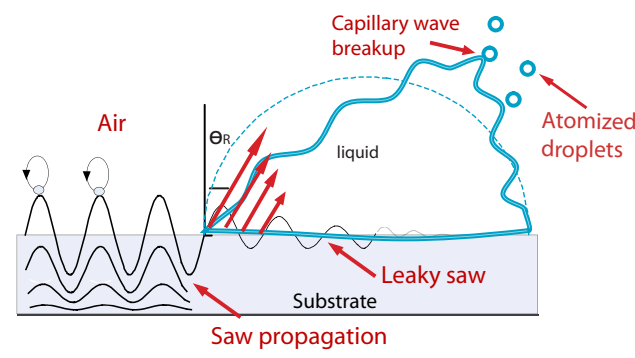

(b)

(c)

Figure 1. (a) A schematic of SAW device shows the IDT electrodes patterned on the piezoelectric $\left(\mathrm{LiNbO}_{3}\right)$ substrate. A RF power source supplies the atomizer with an alternating power via the electrodes and excites the surface acoustic wave (SAW) on the substrate. (b) is an image demonstrates SAW leaks into a liquid and induces the capillary destabilization and breakup, where atomization starts. A photograph of a typical $20 \mathrm{MHz}$ SAW device/atomizer is shown in (c).

makes the SAW atomization capable of delivering the drug particles within required size distribution into the respiratory tract.

\section{EXPERIMENTS AND RESULTS}

\subsection{Drug Solutions}

The aim of this study is to develop a SAW atomizer that can be used for the treatment of asthma, therefore, we employed albuterol as a model drug in our experiments. As albuterol is insoluble in water, but freely dissolved in alcohol, two kinds of alcohol, ethanol and octanol, were used as solvents. Although the toxicity of octanol has not been clearly stated, and its possible ataractic effect should also be carefully noted, ${ }^{19}$ it has been proven to be an efficient therapeutic agent in treating acute pulmonary edema in humans ${ }^{20,21}$ and also a promising compound, in combination with other drugs, to be applied on aerosol therapy for lung related diseases. ${ }^{22}$

\subsection{Aerosol Size Distribution}

Though there are numbers of biophysical parameters that affect the regional deposition of particles/aerosol, ${ }^{23}$ we can only control the aerodynamic particle behavior in the development of the SAW atomizer. Specifically, our focus is on obtaining the optimal aerodynamic size distribution to achieve the maximum deposition of drug particles in the target area. Other parameters, determined by the patients' individual differences $(\mathrm{eg}$. the breathing pattern and airway anatomy), will be considered in the future work when the relevant in-vivo pharmaceutical study is commenced.

There are three modes of deposition in the respiratory airway: the inertia caused impaction, the gravity caused sedimentation and the molecular collision caused diffusion. ${ }^{24}$ The deposition of particles with an aerodynamic diameter greater than $3 \mu \mathrm{m}$ is mainly controlled by the impaction due to their considerable inertial effects, while the sedimentation affects particles bigger than $1 \mu \mathrm{m}$. More specifically, the greater the size is, the higher the possibility is that the aerosols to be deposited in the central airways, or even extrathoracic areas. In contrast, diffusion only affects particles with an aerodynamic size less than $1 \mu \mathrm{m}$. These particles are able to pass through the narrow, tightly curved airways and travel into the deep lung by ideally following the breathing air streams. 
There is also a high possibility that the particles/aerosol that are far less than $0.5 \mu \mathrm{m}$ can also be exhaled by following the exhalation streamlines before they can reach and deposit on the airway walls. Therefore, the particles/droplets deposition positions are closely determined by their aerodynamic sizes at a specified air flow rate. ${ }^{23,25}$

To obtain the aerosol size distribution, we used the laser diffraction based Spraytec (Malvern, UK.) system. The embedded aerosol sizing measurement covers a wide range of particle sizes from 0.1-2000 $\mu \mathrm{m}$, which includes the estimated range $(1-10 \mu \mathrm{m})$ of the aerosol produced by the SAW atomizer.

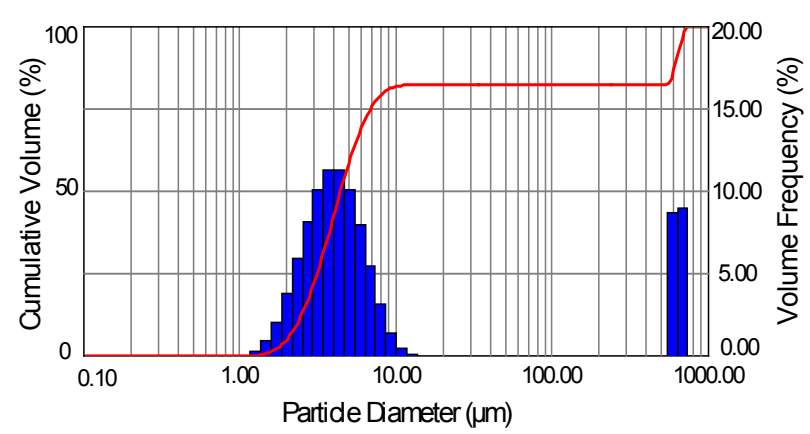

(a)

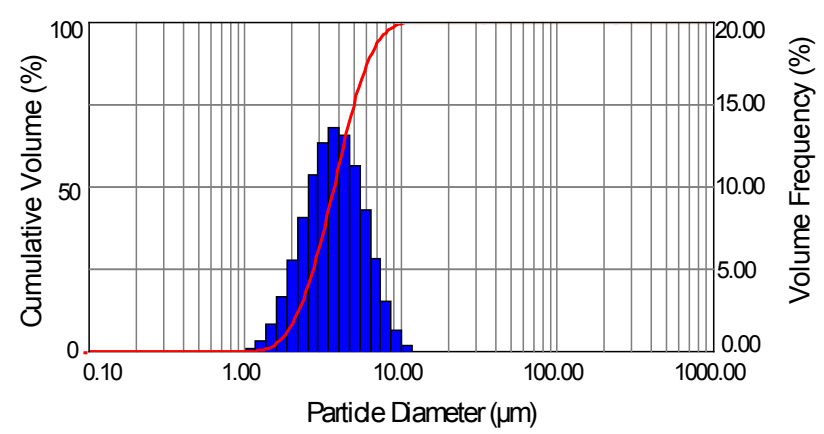

(b)

Figure 2. Albuterol-Octanol atomization: aerosol size distribution acquired by using laser diffraction based Spraytec instrument. (a) The column represents the volume of aerosols within different size bins with respect to the total volume of aerosols detected. The curve is the cumulative volume fraction. By filtering the volume of aerosol with a diameter larger than $100 \mu \mathrm{m}$, the new size distribution shown in (b) is produced. This distribution has an increased percentage of fine particles.

For our measurements, we used octanol as the solvent. The resulting analysis of the size distribution showed a fair agreement between the aerosol size distribution and the deposition requirement, as shown in Fig. 2. In Fig. 2(a), there is a small volume of large droplets, which are greater than $100 \mu \mathrm{m}$ in diameter. These droplets occur due to the relatively larger capillary wavelength, or stronger capillary curvatures, arising when the parent liquid is in a shape of a thick drop instead of a thinner film, as suggested by the equation below:

$$
D \sim \lambda \sim \frac{\gamma H^{2}}{\mu f L^{2}},
$$

where $\lambda$ and $\mu$ are the surface tension and viscosity, respectively, $f$ is the capillary frequency, $H$ and $L$ stand for the liquid film's height and length respectively. ${ }^{18}$ Eq. 1 suggests that a thick drop, with a high height-to-length ratio, will form large droplets. In contrast, a thin liquid film, with a low height-to-length ratio, generates much smaller droplets. This phenomenon can be avoided by applying relatively lower power. If the power is very high, the parent liquid is pushed very quickly by the propagating SAW to the edge of the substrate and forms a conical shaped drop rather than spreading out into a thin film. A group of images shown in Fig. 3 illustrates this phenomenon well. Fig. 3(a) and (b) show the liquid produced higher density monodispersed droplets when the atomization was driven at a relatively low power $(\sim 1.5 \mathrm{~W})$, compared to the power consumption $(>2 \mathrm{~W})$ in the following images, Fig. 3(c) - (f). With low power, there may still be larger jumping drops, for example in Fig. 3(b). However, their occurrence was much less frequent than in the tests that used a higher input power. When the power was increased to approximately $2.2 \mathrm{~W}$, as shown in Fig. 3(c) and (d), the liquid was more likely to form a conical shaped drop and produce larger droplets. From these images, it is easy to distinguish that the smaller droplets were formed out of the liquid film while the larger droplets were pinched off from the conical shaped drop, which had stronger capillary curvatures. When the power was increased to $2.8 \mathrm{~W}$ (Fig. 3(e) and (f)), the trend continued, showing that the large jumping drops appearing more frequently and their sizes also increasing. Due to gravity, the large jumping drops, which are too weighty to be transported into the respiratory tract by the airflow, fall back to the device area, or stop at the entrance of the "mouth". However, it is possible to trap these droplets by using a baffle or a fine mesh, similar to the ones usually employed in the jet-nebulizer. ${ }^{8}$ Therefore, we assumed that droplets with aerodynamic diameter larger than $100 \mu \mathrm{m}$ would be trapped by the 


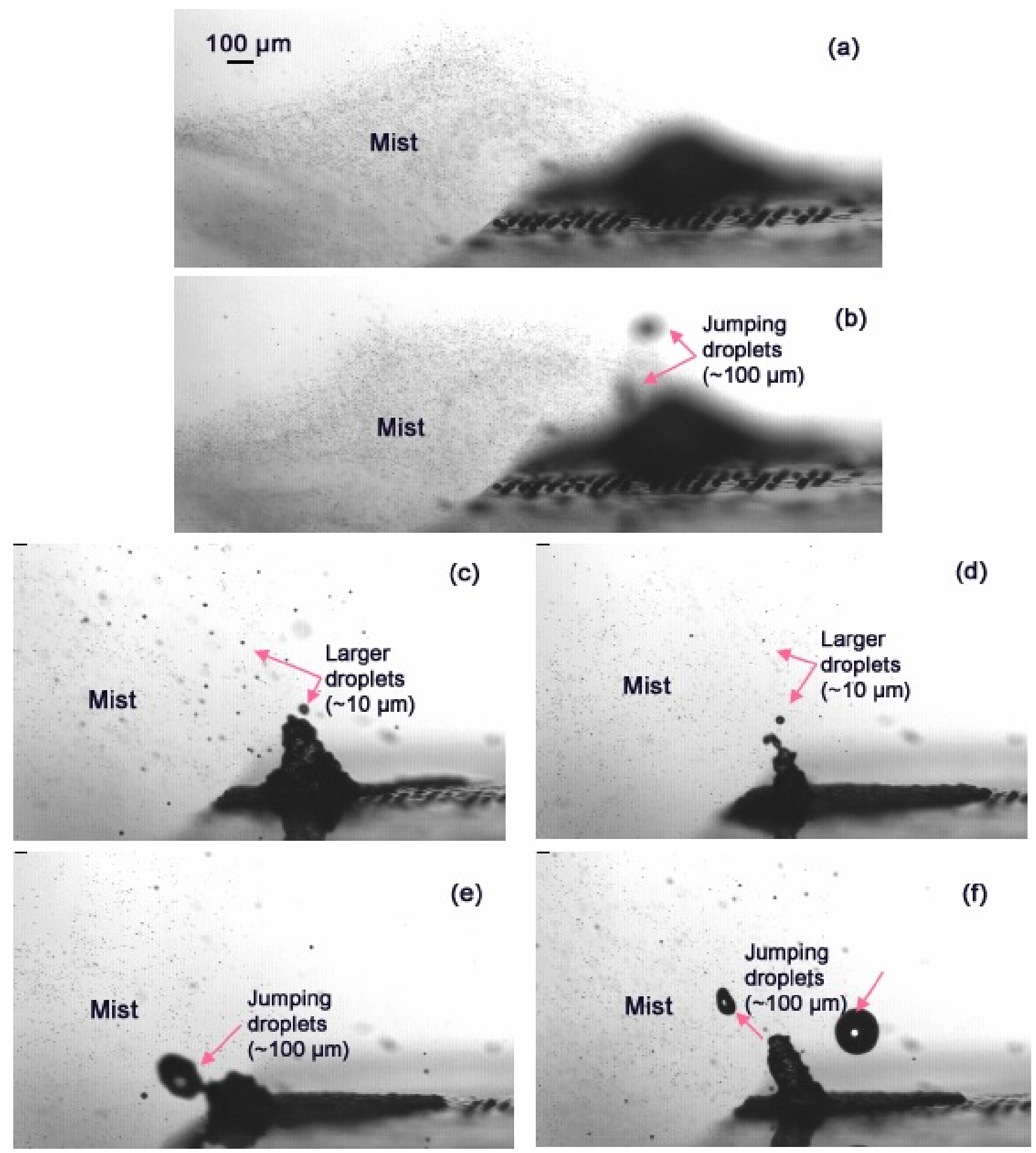

Figure 3. Atomization captured by a high speed camera (3047fps). (a) Atomization is driven at a low power (1.5 W). The image shows the mist is a monodispersed aerosol. Few large jumping droplets appearing at this power. (b) With an increase in power $(2.2 \mathrm{~W})$, a great number of large aerosols appeared $(\sim 10 \mu \mathrm{m})$. (c) With a further increase in power $(2.8$ $\mathrm{W})$, the appearance of large jumping droplets $(\sim 100 \mu \mathrm{m})$ becomes far more frequent. From $(\mathrm{c}) \sim(\mathrm{f})$, the monodispersed aerosol $(\sim 1 \mu \mathrm{m})$, is generated from the liquid film. The liquid used during this experiment is octanol. 
baffle, and filtered the data gained from the Spraytec as shown in Fig. 2(b). According to the computational lung model reported in, ${ }^{23,25}$ within a slow breath pattern — or low air flow rate fashion, for example, $200 \mathrm{ml} / \mathrm{s}$ - the particles with aerodynamic diameters smaller than $7 \mu \mathrm{m}$ have a higher possibility to be deposited in the lung area (bronchi and alveoli), rather than in the extrathoracic area. In comparison, if within a fast breathing pattern, for example $1000 \mathrm{ml} / \mathrm{s}$, the aerodynamic diameter, as a turning point, decreases from $7 \mu \mathrm{m}$ to around $5 \mu \mathrm{m}$. Our results are therefore clearly encouraging - $90 \%$ of the total volume of aerosol produced are in the form of droplets with aerodynamic diameters below $5 \mu \mathrm{m}$, which ensure a high drug deposition in human lung.

From Eq. 1, the aerosol size is also controlled by the ratio of a solution's surface tension and viscosity. Therefore, by replacing the 100\% octanol solvent with a mixture of $50 \%$ octanol and $50 \%$ ethanol, the quantity of droplets with an aerodynamic diameter $>10 \mu \mathrm{m}$ increases. This is confirmed and supported by the results shown in Fig. 4. The latter shows that the ethanol (Fig. 4(a)) produced a lower density of mist and relatively larger droplets than the octanol solution (Fig. 4(b)). Both tests were driven at the same power-1.5 W-on the same atomizer.
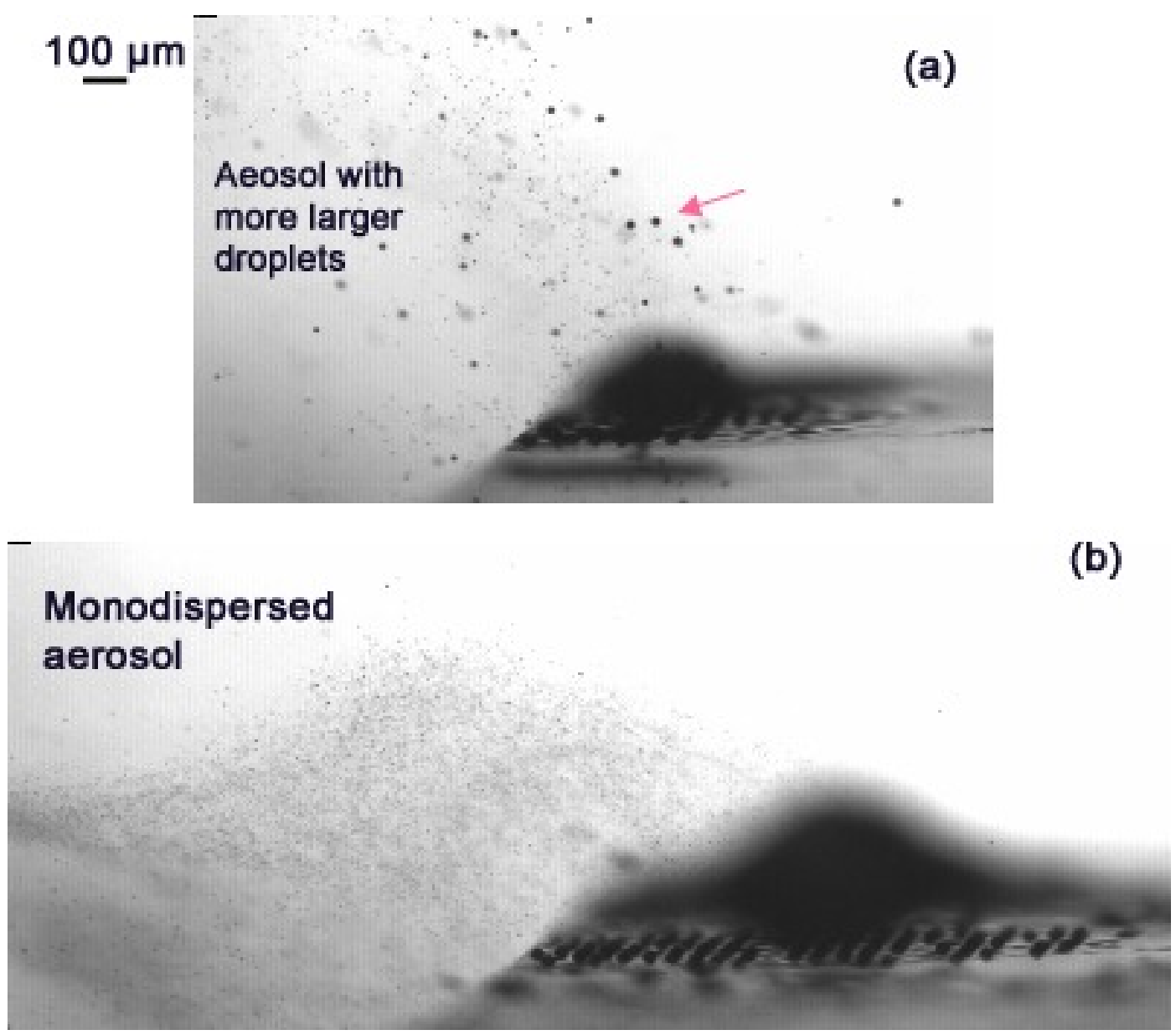

Figure 4. The atomization of albuterol-ethanol solution, shown in (a), produced more larger droplets than albuterol-octanol solution did in (b).

\subsection{The Lung Model and Doses}

\section{The Lung Model}

A one-stage glass twin impinger, was employed to simulate human pulmonary system as a pharmaceutical standard. ${ }^{26}$ As shown in Fig. 5, "A" and "B" represent the oral area and trachea respectively, while the 
bottom glass, "C" (from "C1" to "C2"), is the area that simulates the targeted lung. ${ }^{26-28}$ As albuterol and octanol are immiscible with water, $7 \mathrm{ml}$ and $30 \mathrm{ml}$ of ethanol, instead of water being proposed in, ${ }^{26}$ were placed in "B" and "C" respectively to model the lung humidity. When an airflow of $60 \mathrm{Lmin}^{-1}$ airflow is provided, droplets/particles with an aerodynamic diameter greater than $5.8 \mu \mathrm{m}$ are deposited on the upper airway wall ${ }^{29}$ "A" and "B"-due to the considerable inertial effect. ${ }^{23}$ Droplets/particles with aerodynamic diameter below 5.8 $\mu \mathrm{m}$ are deposited in the lower airways, even deep lung (eg. alveoli), either due to the molecules collision caused diffusion or gravity caused sedimentation. ${ }^{23}$ The amount of particles/aerosol deposited from "C1" to "C2" area is considered as the lung dose, and the total volume of drug solution originally placed upon the atomizer is defined as the theoretical dose.

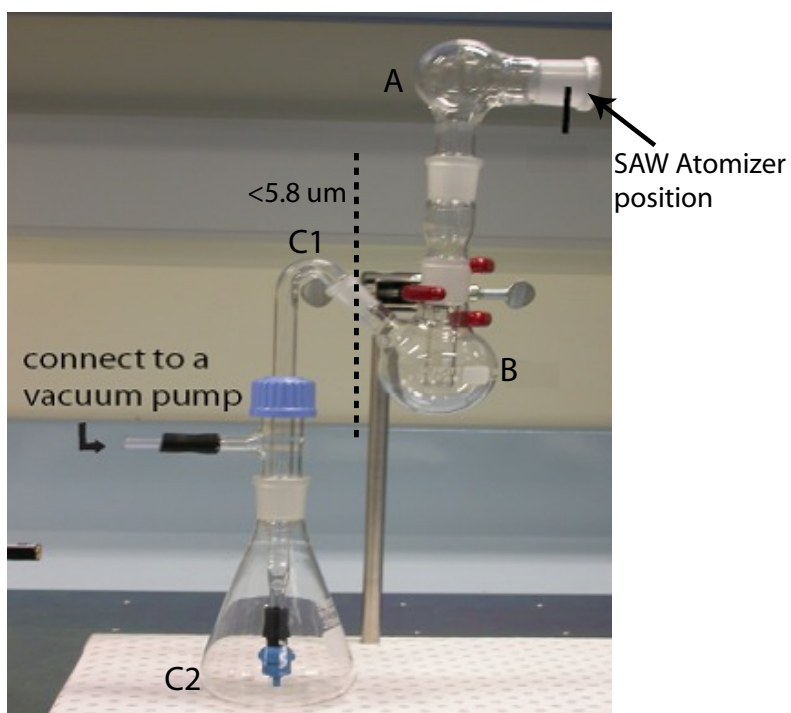

Figure 5. An image shows an ex vivo lung model-a one-stage glass twin impinger-employed in the experiments. A vacuum pump was connected to the impinger at the marked position. A SAW atomizer is placed at the entrance (mouth) of the impinger.

\section{Method and Calibration}

The emitted dose, and the lung dose are the two main factors that must be carefully considered, not only because they are the criterions by which the efficiency of the drug delivery atomizer is judged, but more importantly, are also the guide of pulmonary drug administration for clinical practise purpose. The emitted dose is defined as the true amount of drug solution that is atomized and leaves the atomizer. It equals to the theoretical dose minus the loss on the SAW atomizer. The lung dose stands for the quantity of drug particles that can be transported to the target lung area and absorbed to take effect appropriately. ${ }^{24}$

By comparing the particle amount collected in the "C" area with the theoretical dose placed on the atomizer, we were able to evaluate the lung dose. UV spectrophotometry (Cary 300) was employed to test the drug concentration and obtain drug doses. Fig. 6 is the UV absorption of a group of standard albuterol solutions. It can be seen that albuterol absorbs UV light at $224 \mathrm{~nm}, 278 \mathrm{~nm}$ respectively, with the amount of albuterol linearly related to the UV absorption. We obtained a calibration map as illustrated in the inset, where " $\mathrm{x}$ " represents the drug amount while " $y_{1}$ " and " $y_{2}$ " are the UV absorption at $278 \mathrm{~nm}$ and $224 \mathrm{~nm}$ respectively. We atomized a group of albuterol-octanol solutions and collected the aerosol in each stage of the glass impinger. By applying the UV spectrophotometry on collected solutions from the glass impinger ("A", "B" and "C"), the amount of atomized drugs can be obtained by comparing the absorption values to either of the two linear calibration formulas (" $y_{1}$ " or " $y_{2}$ "). Theoretically speaking, when the UV absorption is known, we can apply either of these two formulas and acquire the same drug concentration. However, during our experiments, suggested by the calibration we obtained, when both peak appeared, applying the absorption data at 278 nm was recommended, since " $y_{1}$ ", with higher value of $R^{2}$, had a smaller fitting error compared to the other, " $y_{2}$ ". In other conditions, 
for example, testing low concentration solutions, of which the absorptions at $278 \mathrm{~nm}$ were too low to be seen, we applied " $y_{2}$ " instead.

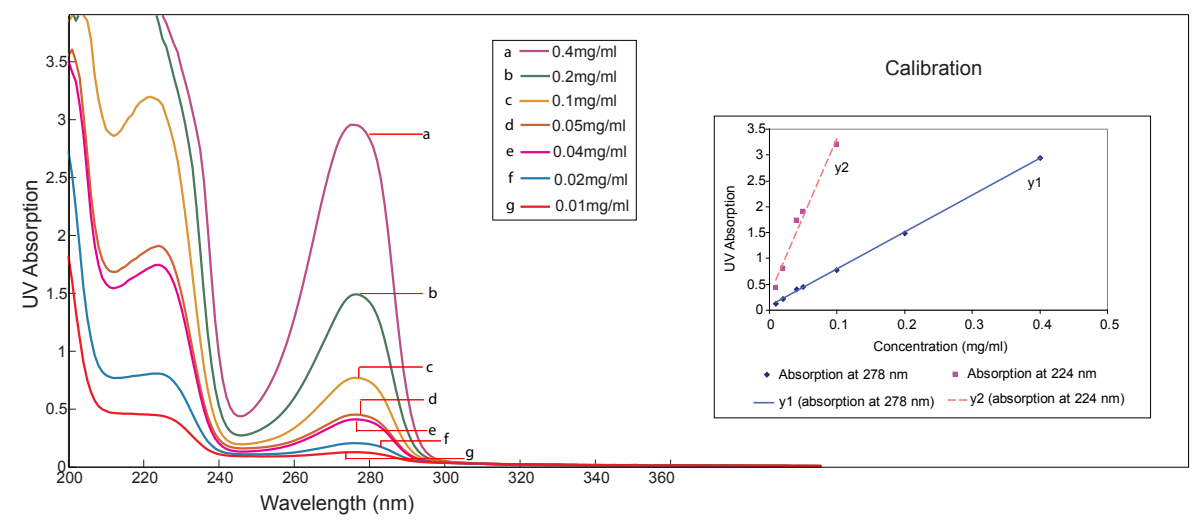

Figure 6. Varied UV absorptions of a group of drug solutions with different albuterol/salbutamol concentrations. It shows there're two peaks of absorption, one peak is around $224 \mathrm{~nm}$ and another is at $278 \mathrm{~nm}$. Inset (right) is the calibration showing the relationship between the UV absorption and albuterol concentration.

\section{The Doses}

Octanol was employed as the solvent as its use was expected to result in a low drug loss. A comparison of atomization using different solvents, such as ethanol and ethanol-water mixture, showed drug loss to be related to the solvent evaporation. As SAWs excite the liquid and destabilize the capillary waves, the liquid spreads into a thin film, as a precursor to atomization commencement. A thin film liquid is easier to atomize than a drop shaped liquid, because less energy will lost due to the damping effect of viscosity. However, the thin film also evaporates very quickly on the other hand. Thus, the most likely situation is that the evaporation of the solvent starts before the atomization commencing, which leaves the solution to become more and more saturated until the drug particles finally precipitated to the atomizer surface. Experimental results revealed that the amount of drugs lost on the atomizer decreased dramatically from more than $80 \%$ to less than $10 \%$ in average, when ethanol was replaced with octanol, a less evaporable fluid. Fig. 7 is a schematic graph showing all the relationships among

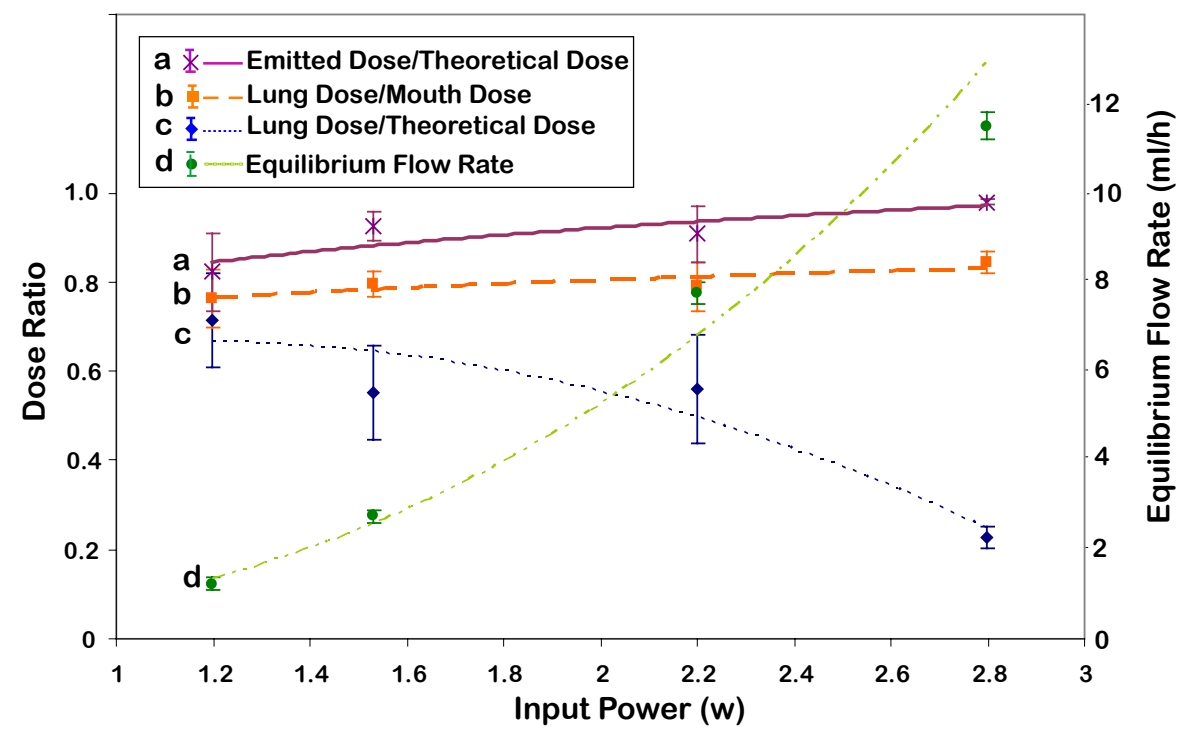

Figure 7. The schematic relationships among the atomization factors, the ratio of different doses to the theoretical dose or mouth dose, the equilibrium flow rate and power. 
the excitation power, equilibrium flow rate, and the ratio of doses with respect to either the theoretical dose or the mouth dose. The equilibrium flow rate, being controlled by an external syringe pump, is not the true atomization rate. The syringe pump provided the atomizer with a continues flow and the speed of which was adjusted to keep the atomization volume on the atomizer constant. The data is shown with error bars and the trendlines are drawn to help visualize the variation trends.

The distance between curve "a" and 100\% horizon stands for the precipitate lost. This, in terms of percentage, is the quantity drug particles left on the atomizer surface with respect to the theoretical amount of drug solution to be atomized. During the atomization, the "emitted dose-to-theoretical dose" ratio, "a", has a stable increase with the increase in power. However, this increasing power results in more jumping drops on the other hand (Fig. 3e-f). Therefore, the "lung deposition-to-theoretical dose" ratio, instead of having a parallel increase as the former, drops sharply with this increase in power, see curve "c". Curve "b", which has a much slighter increase, when compared to "a", represents the ratio of the lung dose to the mouth dose, and the latter equals to the emitted dose minuses the total volume of the large jumping drops. Even though the effect from the jumping drops has been excluded, comparing "a", the flatter slope of "b" means the lung deposition does not grow equally to the emitted dose, which can be explained by the phenomena shown in (Fig. 3c-f). These pictures clearly show that the higher power helps to increase the emitted dose and equilibrium flow rate ("d") on one hand, but introduces a higher probability of inducing stronger capillary curvatures on the other hand. These strong curvatures result in more large droplets $(\sim 10 \mu \mathrm{m})$ pinched off and, in turn, produces relatively less fine particles/droplets.

\section{CONCLUSION}

SAW atomization has been shown to be a promising technology to be applied on inhaled pulmonary drug delivery system as a potential asthma nebulizer. The SAW atomizer has demonstrated advantages in many aspects, including a small scale and low power requirements. More importantly, it is able to efficiently produce monodispersed aerosol with the required size distribution for useful asthma treatment. In this paper, both the images captured from a high speed camera, and the results obtained from the laser diffraction based Spraytec technology, show the aerosol produced by the SAW device to be within the required range, and that the particle size can be controlled with appropriate excitation power and solution properties. In addition, a one-stage twin impinger was employed as a lung model and demonstrated a high drug deposition in the "lung" area, proving the excellent efficiency from the SAW excited atomization to be applied on pulmonary aerosol therapy.

\section{REFERENCES}

[1] B. H. J. Dickhoff, Adhesive mixtures for powder inhalation. PhD thesis, University of Groningen, 2006.

[2] P. J. Barnes, I. W. Rodger, and N. C. Thomson, eds., Asthma: Basic Mechanisms and Clinical Management, Elsevier Ltd., 1998.

[3] H. Frijlink and A. Boer, "Dry powder inhalers for pulmonary drug delivery," Expert Opinion on Drug Delivery 1, pp. 67-86, 2004.

[4] N. Carroll, C. Cooke, and A. James, "The distribution of eosinophils and lymphocytes in the large and small airways of asthmatics," European Respiratory Journal 10, pp. 292-300, 1997.

[5] H. Chrystyn, "Is total dose more important than particle distribution," Respiratory Medicine 91, pp. 17-19, 1997.

[6] S. Pedersen and O. OByrne, "A comparison of the efficacy and safety of inhaled corticosteroids in asthma," Allergy 52, pp. 1-34, 1997.

[7] L. Gradon and J. Marijnissen, eds., Optimization of Aerosol Drug Delivery, Kluwer Academic Publishers, 2003.

[8] R. Dalbya and J. Suman, "Inhalation therapy: technological milestones in asthma treatment," Advanced Drug Delivery Reviews 55, pp. 779-791, 2003.

[9] M. Cloupeau and B. Prunet-Foch, "Electrohydrodynamic spraying functioning modes: a critical review," Journal of Aerosol Science 25, pp. 1021-1036, 1994. 
[10] J. Grace and J. Marijnissen, "A review of liquid atomization by electric means," Journal of Aerosol Science 25, pp. 1005-1019, 1994.

[11] J. C. Ijsebaert, K. B. Gerse, J. C. M. Marijnissen, J.-W. J. Lamers, and P. Zanen, "Electro-hydrodynamic atomization of drug solutions for inhalation purposes," Journal of Applied Physiology 91, pp. 2735-2741, 2001.

[12] G. Macchiarella and G. B. Stracca, "Saw device for telecommunications: examples and applications," in Ultrasonics Symposium, 1982.

[13] E. P. Papadakis, Ultrasonic Instruments and Devices: Reference for Modern Instrumentation, Techniques, and Technology, Academic Press, 1999.

[14] M. Kurosawa, A. Futami, and T. Higuchi, "Characteristics of liquids atomization using surface acoustic wave," in Proceedings of Transducer 97, Chicago, USA, 1997.

[15] J. Friend and L. Yeo, "Observation and analysis of surface acoustic wave induced atomization," in Proceedings of the 5th ACAM 2007, Brisbane, Australia, 2007.

[16] H. Li, J. Friend, and L. Yeo, "Surface acoustic wave concentration of particle and bioparticle suspensions," Biomedical Microdevices 28, pp. 4098-4104, 2007.

[17] R. Shilton, M. K. Tan, L. Y. Yeo, and J. R. Friend, "Particle concentration and mixing in microdrops driven by focused surface acoustic waves," Journal of Applied Physics 104, p. 014910, 2008.

[18] A. Qi, L. Yeo, and J. Friend, "Interfacial destabilization and atomization driven by surface acoustic waves," Physics of Fluids 20, p. 074103, 2008.

[19] C. M. Sinton, B. I. Krosser, K. D. Walton, and R. R. Llinas, "The effectiveness of different isomers of octanol as blockers of harmaline-induced tremor," European Journal of Physiology 414, pp. 31-36, 1989.

[20] N. E. Reich, B. A. Rosenberg, and M. Metz, "The use of 2-ethylhexanol in acute pulmonary edema," Diseases of the Chest 22, pp. 43-49, 1953.

[21] W. F. Miller, "Aerosol therapy in acute and chronic respiratory disease," Archives of Internal Medicine 131, pp. 148-155, 1973.

[22] B. Marcet, F. Becq, C. Norez, P. Delmas, and B. Verrier, "General anesthetic octanol and related compounds activate wild-type and delf508 cystic fibrosis chloride channels," British Journal of Pharmacology 141, pp. 905-914, 2004.

[23] G. Scheuch, M. J.Kohlhaeufl, P. Brand, and R. Siekmeier, "Clinical perspectives on pulmonary systemic and macromolecular delivery," Advanced Drug Delivery Reviews 58, pp. 996-1008, 2006.

[24] R. Gregory, Evaluation of the nebulization function of the intrapulmonary percussive ventilation. $\mathrm{PhD}$ thesis, Universite Catholique de Louvain, 2006.

[25] I. C. on Radiological Protection, "Human respiratory model for radiological protection (icrp publication 66)," Ann. ICRP 24, pp. 1-120, 1994.

[26] "Aerodynamic assessment of fine particles," British Pharmacopoeia Appendix XII F, p. A203, 1999.

[27] T. Srichana, "A human oral throat cast integrated with a twin-stage impinger," Journal of Pharmacy and Pharmacology 52, pp. 771-778, 2000.

[28] C. M. Vozone and H. M. C. Marques, "Complexation of budesonide in cyclodextrins and particle aerodynamic characterization of the complex solid form for dry powder inhalation," Journal of Inclusion Phenomena and Macrocyclic Chemistry 44, pp. 111-115, 2000.

[29] C. M. Vozone and H. M. C. Marques, "Complexation of budesonide in cyclodextrins and particle aerodynamic characterization of the complex solid form for dry powder inhalation," Journal of Inclusion Phenomena and Macrocyclic Chemistry 44, pp. 111-115, 2002. 